\title{
Talmudic Approach to Load Shedding of Islanded Microgrid Operation Based on Multiagent System
}

\author{
Hak-Man Kim*, Tetsuo Kinoshita** and Yujin Lim $^{\dagger}$
}

\begin{abstract}
This paper presents a load-shedding scheme using the Talmud rule in islanded microgrid operation based on a multiagent system. Load shedding is an intentional load reduction to meet a power balance between supply and demand when supply shortages occur. The Talmud rule originating from the Talmud literature has been used in bankruptcy problems of finance, economics, and communications. This paper approaches the load-shedding problem as a bankruptcy problem. A load-shedding scheme is mathematically expressed based on the Talmud rule. For experiment of this approach, a multiagent system is constructed to operate test islanded microgrids autonomously. The suggested loadshedding scheme is tested on the test islanded microgrids based on the multiagent system. Results of the tests are discussed.
\end{abstract}

Keywords: Microgrid, Multiagent system, Load shedding, Talmud rule, Bankruptcy problem

\section{Introduction}

Currently, interest in the microgrid, an eco-friendly small-scale power system, has increased. The microgrid is a subsystem of the Smart Grid, and is composed of distributed generation systems (DGs), distributed storage devices (DSs), and loads such as residential buildings, commercial buildings, schools, hospitals, industrial plants, and so on.

The microgrid can be operated in two modes: gridconnected and islanded. In the grid-connected mode, a power balance between supply and demand can be maintained by power exchange with the power grid. In contrast, maintaining the balance is a very important problem in islanded operation because there is no power exchange with any power grids. In the case of supply shortage, load shedding, which is a control action to reduce amounts of load intentionally, and the charging action of DSs are used to meet the balance. Load shedding is a critical problem because it makes consumers uncomfortable. Load shedding is performed by the order of low priority from the viewpoint of load importance in one-owner systems. Furthermore, this scheme is widely used in existing power systems. However, in autonomous multiparticipant systems, the scheme is not effective for load shedding because of competing and conflicting claims of consumers for their loads. Therefore, an effective scheme for load shedding is required in islanded microgrid operation based on autonomous multiparticipants.

Recently, multiagent systems have been studied for

$\dagger \quad$ Corresponding Author: Dept. of Information Media, Univ. of Suwon, Hwaseong, Korea (yujin@suwon.ac.kr)

* Dept. of Electrical Engineering, University of Incheon, Incheon, Korea (hmkim@incheon.ac.kr)

** Research Institute of Electrical Communication, Graduate Schoo of Information Science, Tohoku University, Sendai, Japan (kino@ riec.tohoku.ac.jp)

Received: July 27, 2010; Accepted: January 12, 2011 autonomous operation of microgrids [1-4]. An agent (or intelligent agent) can perceive its environment, can make a decision against changes of the environment, and can act to resolve them autonomously according to its design purpose using its reactivity, proactiveness, and social ability [5].

The bankruptcy problem divides an insufficient estate among all claimants. Historically, the bankruptcy problem originates from the Talmud literature. Two well-known stories are related to the bankruptcy problem in the Talmud literature: the contested garment problem and the estate division problem. Solutions of the stories are based on a rule: the Talmud rule. The principle of the Talmud rule was first mathematically explained by Aumann and Maschler in 1985. Since then, the Talmud rule has been used for many bankruptcy problems in finance, economics, communications, and so on [6-9].

In this paper, the load-shedding problem is approached as a bankruptcy problem. A load-shedding scheme mathematically formulated based on the Talmud rule is suggested. A multiagent system for islanded microgrid operation is constructed. The proposed load-shedding scheme is tested on sample islanded microgrids based on the multiagent system to show the feasibility, after which the test results are discussed.

\section{Islanded Microgrid Operation}

Fig. 1 shows the configuration of the microgrid. In the figure, the microgrid operation and control center (MGOCC) has important functions related to operating the microgrid, controlling and monitoring protection devices, and communicating with participants of the microgrid. In addition, the MGOCC exchanges information with the power grid in the grid-connected mode [3]. 


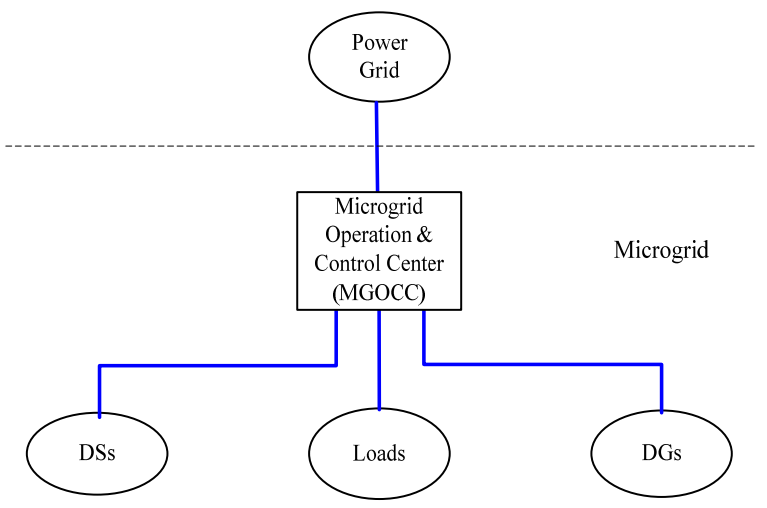

Fig. 1. Microgrid configuration [3].

The microgrid can be electrically isolated from a power grid by abnormal conditions, such as fault occurrence at the power grid, or by geographical isolation, such as a distant island. This is called an islanded microgrid. To meet a power balance between supply and demand in the microgrid, the following actions are generally performed:

- Decrease in generation and the charge action of DS for solving power supply surplus;

- Load shedding and the discharge action of DS for solving power supply shortage.

Load shedding is a critical problem because of competing and conflicting claims of consumers resulting from inconvenience. Fig. 2 shows the typical operation scheme according to a power imbalance in the islanded microgrid.

In this study, MGOCC should establish an operation plan for the next interval, and should implement an operation plan established during the previous interval (Fig. 3), similar to the operation of power grids [3]. In addition, the MOGCC should solve the load-shedding problems whenever supply shortages occur in the islanded mode.

\section{Load-shedding Scheme Based on the Talmud Rule}

\subsection{Bankruptcy Problem}

A bankruptcy problem is defined as a pair $(c, E)$, where $E$ is an amount to be divided and $c=\left(c_{1}, \ldots, c_{N}\right)$ is a set of claims of $N$ agents, which is described as

$$
0 \leq c_{1} \leq \ldots \leq c_{\mathrm{N}} \text { and } 0 \leq E \leq c_{1}+\ldots+c_{\mathrm{N}} .
$$

A solution of a bankruptcy problem is a vector of real numbers, $x=\left(x_{1}, \ldots, x_{N}\right)$ with $x_{1}+\ldots+x_{N}=E$., where $x_{i}$ the amount assigned to claimant $c_{i}$ [9].

\subsection{Talmud Rule}

The Talmud rule is widely used in bankruptcy problems

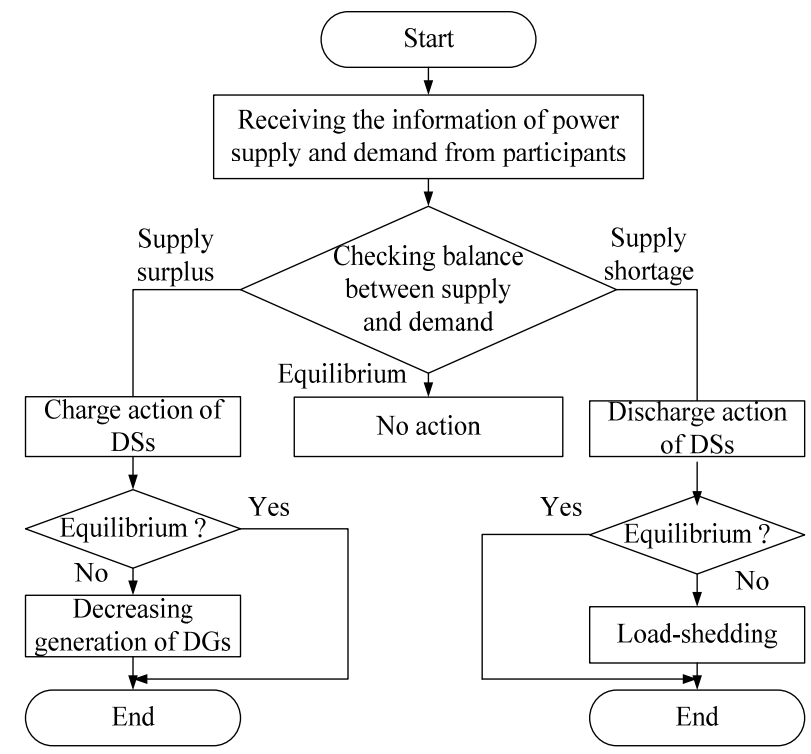

Fig. 2. Typical operation scheme according to power imbalance.

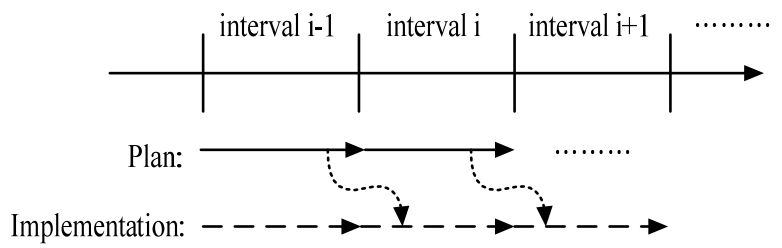

Fig. 3. Microgrid operation procedure [3].

Table 1. Results of the estate division problem

\begin{tabular}{cccc}
\hline Estateldebt & 100 & 200 & 300 \\
\hline 100 & $33^{1 / 3}$ & $33^{1 / 3}$ & $33^{1 / 3}$ \\
200 & 50 & 75 & 75 \\
300 & 50 & 100 & 150 \\
\hline
\end{tabular}

of many areas such as finance, economics, and communications. Table 1 shows the result of a well-known estate division problem in the Babylonian Talmud literature [9].

The following theorem [6] shows that the Talmud rule gives a unique solution for a bankruptcy problem:

Theorem) Each bankruptcy problem has a unique consistent solution.

\subsection{Intuitive Insight from the Talmud Rule}

For intuitive insight of the basic mechanism of the Talmud rule, Fig. 4 [9], which shows results of the estate division problem according to expansion of estate until total claim, is used.

The following basic mechanism of the Talmud rule can be seen in Fig. 4:

- Interval A: Until the smallest claimant arrives at half of 


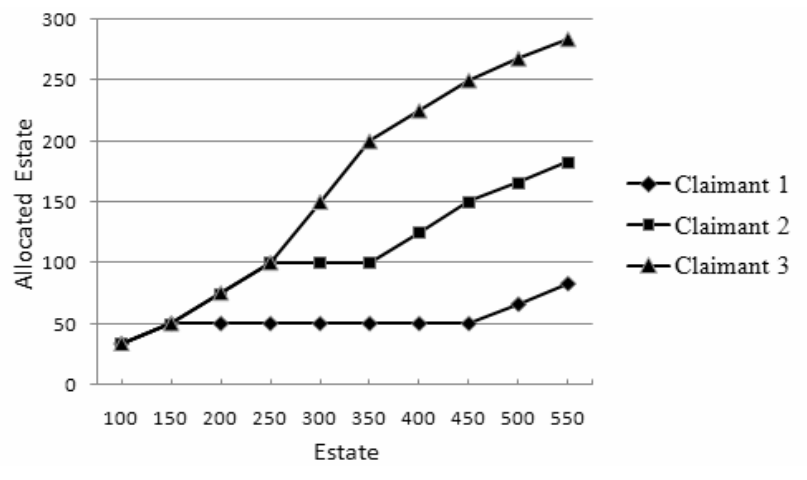

Fig. 4. Result of the estate division problem.

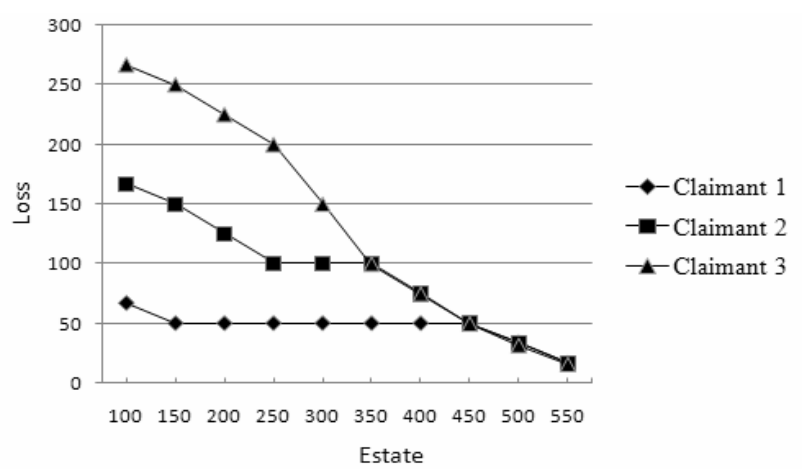

Fig. 5. Loss of each claimant.

his/her claim, the estate is equally divided to every claimant $(0<$ estate $\leq 150)$.

- Interval B: Until the biggest claimant arrives as half of his/her claim, the estate is equally divided to other claimants excluding some claimants arriving at halves of their claims $(150<$ estate $\leq 300)$.

- Interval C: Until the smallest claimant joins in division again, the estate is equally divided with a similar pattern of Interval B $(300<$ estate $\leq 450)$.

- Interval D: Estate is near proportional to claim (450 > estate).

To clarify the above-mentioned consideration, losses of claimants of the estate division problem are shown in Fig. 5. A near-proportional relationship in Interval D is based on equal loss of each claimant. In addition, a duality between allocated estate and loss in the Talmud rule. A previous study [6] has explained the duality briefly. Therefore, the loss of each claimant has a unique solution because it is decided by a unique solution of the Talmud rule. The loadshedding problem is related to the loss of each claimant in the Talmudic example.

\subsection{Load-shedding Scheme Based on Talmud Rule}

Load shedding is a control action that intentionally reduces loads to meet a power balance between supply and demand when supply shortages occur in the islanded mode. Load shedding causes consumers inconvenience and therefore should be performed in a rule.
The load-shedding problem is expressed as a bankruptcy problem. The load-shedding problem can be defined as a pair $(l, P)$, where $P$ is available power and $l=\left(l_{1}, \ldots, l_{n}\right)$ is the vector of claims of loads, which is described as

$$
\leq l_{1} \leq \ldots \leq l_{n} \text { and } 0 \leq P \leq l_{1}+\ldots+l_{n} \text {. }
$$

The vector of allocated power $\left(l^{*}\right)$ of each load is defined as

$$
l^{*}=\left(l_{1}^{*}, \ldots, l_{n}^{*}\right)
$$

and the Talmud rule for allocating load is defined by Eqs. (4) and (5):

$$
\text { If } \Sigma\left(l_{i} / 2\right) \geq P, \quad T_{i}(l, P)=\min \left\{l_{i} / 2, \lambda\right\},
$$

where $\lambda$ is chosen so that $\Sigma \min \left\{l_{i} / 2, \lambda\right\}=P$.

$$
\text { If } \Sigma\left(l_{i} / 2\right) \leq P, \quad T_{i}(l, P)=l_{i}-\min \left\{l_{i} / 2, \lambda\right\},
$$

where $\lambda$ is chosen so that $\Sigma\left[l_{i}-\min \left\{l_{i} / 2, \lambda\right\}\right]=P$.

Finally, the vector of load-shedding amount of each load $\left(s^{*}\right)$ is calculated by Eq. (6):

$$
s^{*}=\left(s_{1}^{*}, \ldots, s_{\mathrm{n}}^{*}\right)=l-l^{*} .
$$

From Table 1, an infinite decimal can be the result, which is unacceptable in the practical application. For this, the following additional computation rule is considered:

(Computation Rule) Amount below the decimal point is truncated and the amount is allocated to a consumer requiring the largest load.

\section{Building Multiagent System for Islanded Microgrid}

\subsection{Background}

An agent is anything that can perceive its environment through sensors and act upon that environment through actuators [10]. For communications among agents, messages are sent using the agent communication language (ACL). In this paper, a modified version of the knowledge query and manipulation language as the ACL is used. A message format and a template format used in the proposed multiagent system, respectively, are shown below [11]:

$$
\begin{gathered}
\text { (<performative }>\text { : from }<\text { agent name }>\text { : to }<\text { agent name }> \\
\text { : content }<\text { OAV type data }>\text { ) } \\
\text { (template name: ID identifier OAV type data list) }
\end{gathered}
$$

where OAV type data are composed of an object, an attribute of the object, and the value of the attribute.

As communication links, the Internet, power line communication (PLC), fiber optic lines, leased lines, and wire- 
less communications, and so on, can be used. The detailed communication links are not included in this paper.

As a protocol for interactions among agents, the contract net protocol (CNP) is widely used. The CNP was developed to solve cooperative distributed problems in the communication areas by R.G. Smith, and is the most used protocol for interactions among agents $[5,12]$. The following shows the basic steps of the CNP [13]:

Step 1: A manager announces the existence of a task via a broadcast message.

Step 2: Agents evaluate the announcement, and several of these agents having the solving capability against the task submit a bid.

Step 3: The manager awards a contract to the most suitable agent among candidate agents as a contractor for that task.

For the effective task management, the following state transition function $(T)$ is used [11]:

$$
\left(s^{\prime}, a\right)=T(s, e)
$$

Many tools have been developed to build multiagent systems. In this paper, a Distributed Agent System based on Hybrid architecture (DASH) as a multiagent platform, Interactive Design Environment for Agent system (IDEA) as a GUI-based interactive environment for the DASH platform, and Java for writing user-defined functions are used [14-16].

\subsection{Building Multiagent System}

To construct a multiagent system to operate an islanded microgrid autonomously, a multiagent system for islanded microgrid operation is defined as

$$
A g=\left\{A g_{\mathrm{MGOCC}}, A G_{\mathrm{DG}}, A G_{\mathrm{DS}}, A G_{\mathrm{L}}\right\},
$$

where $A g_{\text {MGOCC }}$ is the MGOCC agent, $A G_{\mathrm{L}}$ is a set of load agents $\left(A g_{\mathrm{L}}\right), A G_{\mathrm{DG}}$ is a set of DG agents $\left(A g_{\mathrm{DG}}\right)$, and $A G_{\mathrm{DS}}$ is a set of storage device agents $\left(A g_{\mathrm{DS}}\right)$. The main functions of the agents are defined as follows:

- $A g_{\text {MGOCC }}$ has sufficient knowledge and information as a manager for microgrid operation.

- $A g_{\mathrm{DG}}$ governs a DG or a group of DGs located at the same place.

- $A g_{\mathrm{DS}}$ takes charge of a storage devices or a group of storage devices located at the same place.

- $A g_{\mathrm{L}}$ operates and controls its load including load shedding.

Fig. 6 shows the basic structure of the above-mentioned multiagent system for islanded microgrid operation.

Fig. 7 shows a designed message flow among the agents for cooperative distributed problem solving. For message

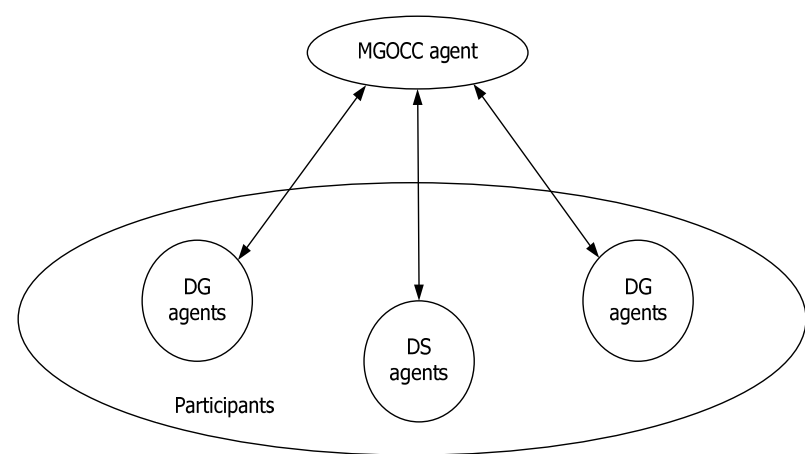

Fig. 6. Basic structure of a multiagent system for islanded microgrid operation.

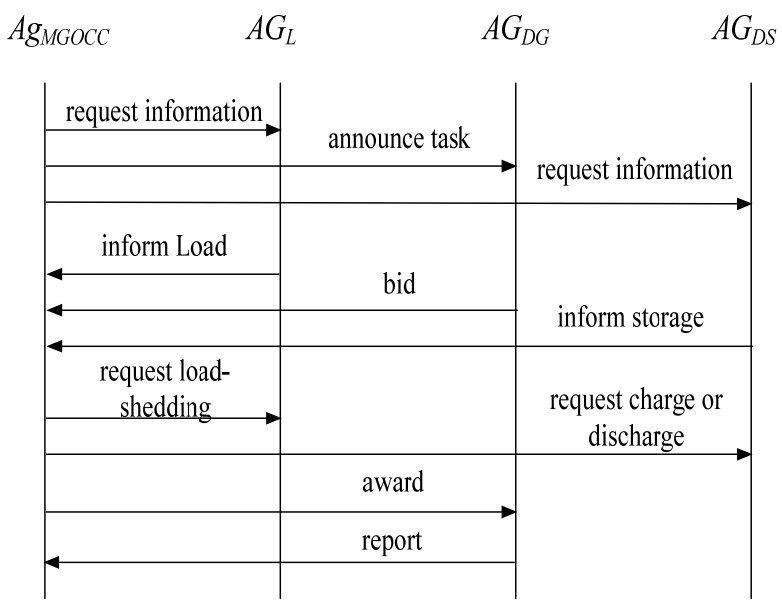

Fig. 7. Message flow among agents.

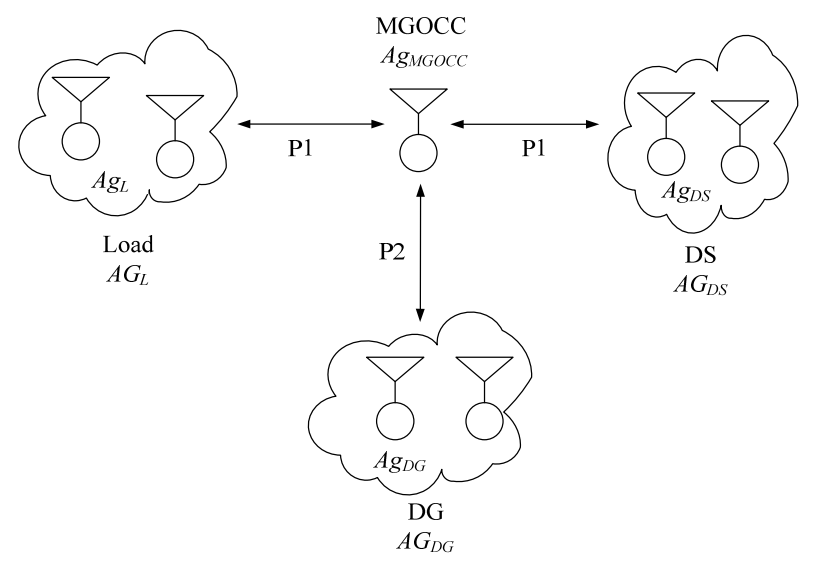

P1: Information Exchange Protocol

P2: Modified CNP

Fig. 8. Protocols among agents.

exchange in Fig. 7, an information exchange protocol for interactions between $A g_{\mathrm{MGOCC}}$ and $A G_{\mathrm{DS}} / A G_{L}$, and a modified CNP for interactions between $A g_{\mathrm{MGOCC}}$ and $A G_{\mathrm{DG}}$ are designed (Fig. 8). The modified CNP is based on the basic steps of the CNP [11-12], but is tailored appropriately for microgrid operation. 


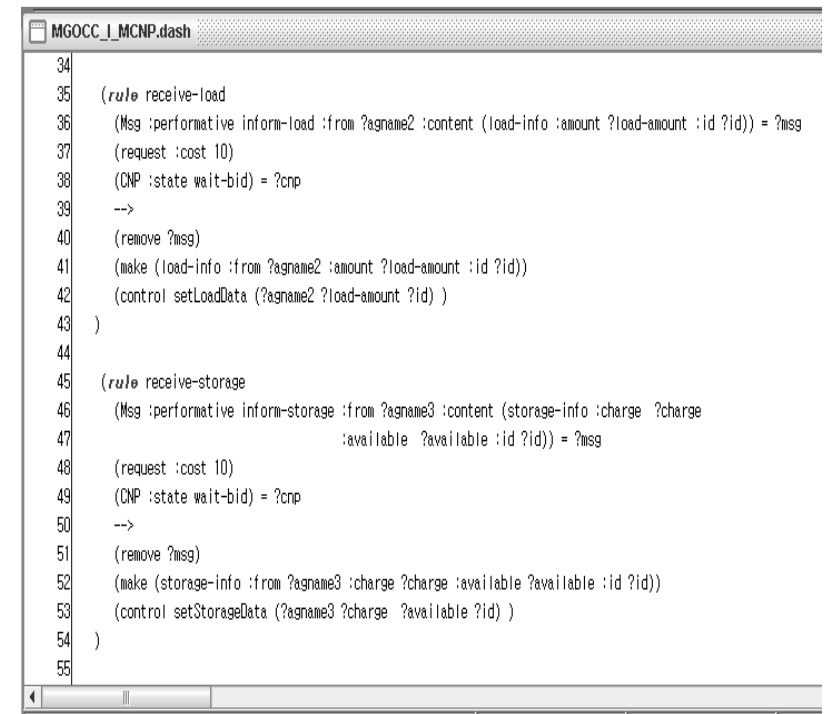

Fig. 9. Part of the MGOCC agent programmed on IEDA.

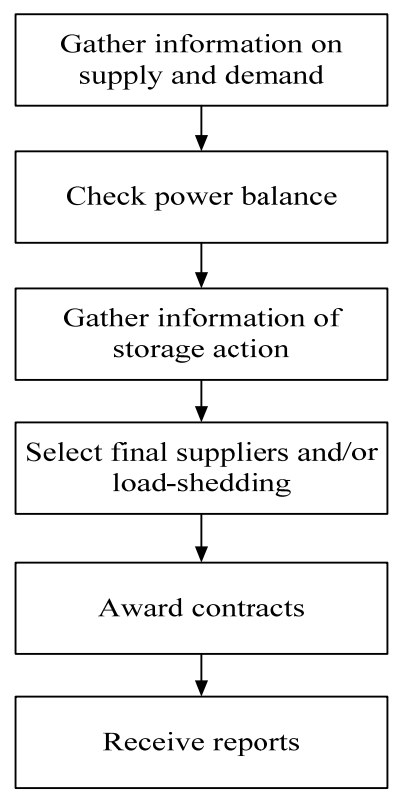

Fig. 10. $A g_{\mathrm{MGOCC}}$ workflow.

Fig. 9 shows a part of the MGOCC agent programmed on the edit window of IDEA environment.

The major features of the proposed multiagent system constructed for islanded microgrid operation are as follows:

- $A g_{\mathrm{MGOCC}}$ is a manager of islanded operation. Fig. 10 shows the $A g_{\text {MGOCC }}$ workflow.

- DGs submit production costs as bid prices.

- The final contractors among DGs are decided by the merit order.

- The strategy and priority for decision-making of $A g_{\text {MGOCC }}$ are designed to follow along the typical operation procedure, as shown in Fig. 2.

- In the case of supply shortage, $A g_{\mathrm{MGOCC}}$ decides load

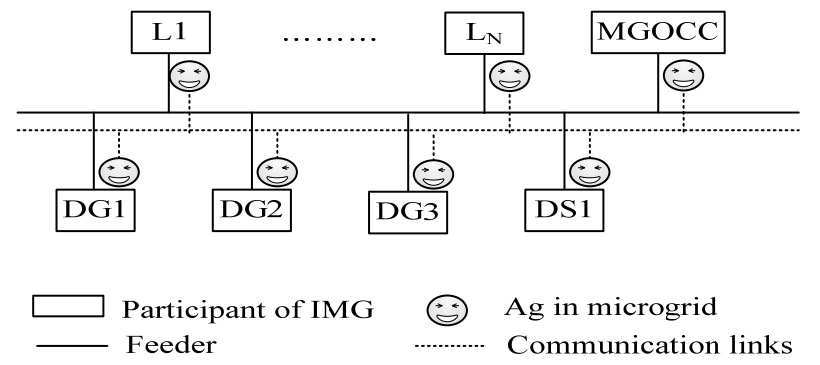

Fig. 11. Configuration of an agent-based islanded microgrid for Tests 1 and 2.

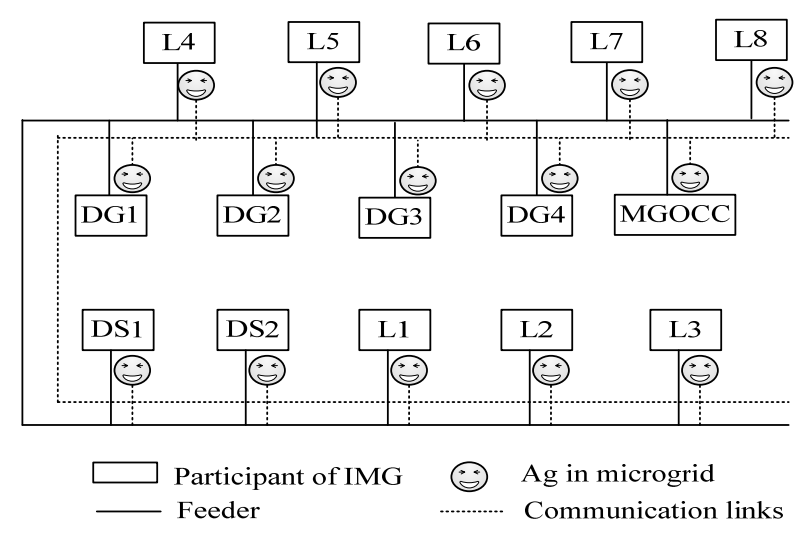

Fig. 12. Configuration of an agent-based islanded microgrid for Test 3 .

shedding by the load-shedding scheme mentioned in Section 3.4.

- The agent-based islanded microgrid is implemented under the Windows XP operating system.

\section{Experiment}

To test the proposed load-shedding scheme in the case of supply shortage, two agent-based islanded microgrids are constructed, as shown in Figs. 11 and 12, where IMG means the islanded microgrid. In Fig. 11, a multiagent system is composed of an MGOCC agent $\left(A g_{\text {MGOCC }}\right)$, three DG agents ( $\left.A g_{\mathrm{DG} 1}, A g_{\mathrm{DG} 2}, A g_{\mathrm{DG} 3}\right)$, a DS agent $\left(A g_{\mathrm{DS} 1}\right)$, and $N$ load agents $\left(A g_{\mathrm{L} 1}, \ldots, A g_{\mathrm{LN}}\right)$ to test general features of the Talmud rule-based load-shedding scheme (Tests 1 and 2). In Fig. 12, a multiagent system is composed of an MGOCC agent $\left(A g_{\mathrm{MGOCC}}\right)$, four DG agents $\left(A g_{\mathrm{DG} 1}, A g_{\mathrm{DG} 2}\right.$, $\left.A g_{\mathrm{DG} 3}, A g_{\mathrm{DG} 4}\right)$, two DS agents $\left(A g_{\mathrm{DS} 1}, A g_{\mathrm{DS} 2}\right)$, and eight load agents $\left(A g_{\mathrm{L} 1}, \ldots, A g_{\mathrm{L} 8}\right)$ to test islanded microgrid operation (Test 3).

Test 1 checks whether the proposed load-shedding scheme based on the Talmud rule works well in the proposed agent-based microgrid with 10 load agents. In Test 2, the processing time for solving load shedding according to the number of load agents is checked to test the feasibility of application in fields. Test 3 checks an emergency condition by a sudden fault of a DG (DG2 in Fig. 12) after estab- 


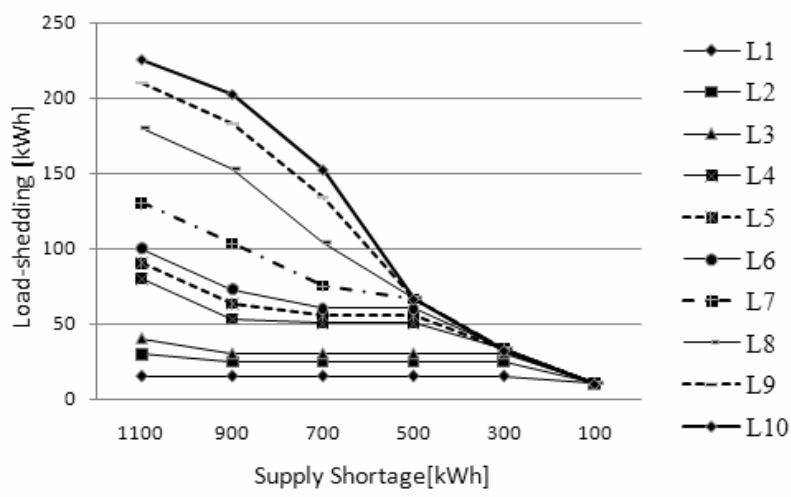

Fig. 13. Load-shedding result of Test 1 .

lishing an operation plan for the next interval, where load shedding will be considered to solve a power imbalance according to out-of-service DG2.

\subsection{Test 1}

For test agent-based islanded microgrid operation, 10 loads are considered. The number of agents is 15 . Information of a DS (DS1) and 10 loads of agent-based islanded microgrid shown in Fig. 11 are as follows:

- Capacity of DS1: $10 \mathrm{kWh}$

- Initial charged amount of DS1: $5 \mathrm{kWh}$

- L1: $30 \mathrm{kWh}$

- L2: $50 \mathrm{kWh}$

- L3: $60 \mathrm{kWh}$

- L4: $100 \mathrm{kWh}$

- L5: $110 \mathrm{kWh}$

- L6: $120 \mathrm{kWh}$

- L7: $150 \mathrm{kWh}$

- L8: $200 \mathrm{kWh}$

- L9: $230 \mathrm{kWh}$

- L10: $250 \mathrm{kWh}$

Supply shortage is given from 1100 to $100 \mathrm{kWh}$ and decrease by $200 \mathrm{kWh}$ in steps. For this, suitable capacities of three DGs are allocated in six cases according to required load amount. Fig. 13 shows the result of Test 1 . The pattern of load shedding according to amounts of supply shortage shows the Talmudic pattern of Fig. 5. Clearly, the proposed load-shedding scheme works well in the proposed agentbased microgrid operation.

\subsection{Test 2}

Processing time for solving load shedding is checked according to the number of load agents to evaluate the feasibility for applying fields. Estimation of the processing time is based on the process depicted in Fig. 10. The processing time of Steps 1,3 , and 5 are mainly determined by the messaging overheads between the MGOCC and DG/L agents, which are proportional to the number of agents. In contrast, the processing time of Steps 2, 4, and 6 are de-
Table 2. Results of the estate division problem

\begin{tabular}{c|c|c}
\hline Case & Number of loads & Number of agents \\
\hline 1 & 10 & 15 \\
\hline 2 & 20 & 25 \\
\hline 3 & 30 & 35 \\
\hline
\end{tabular}

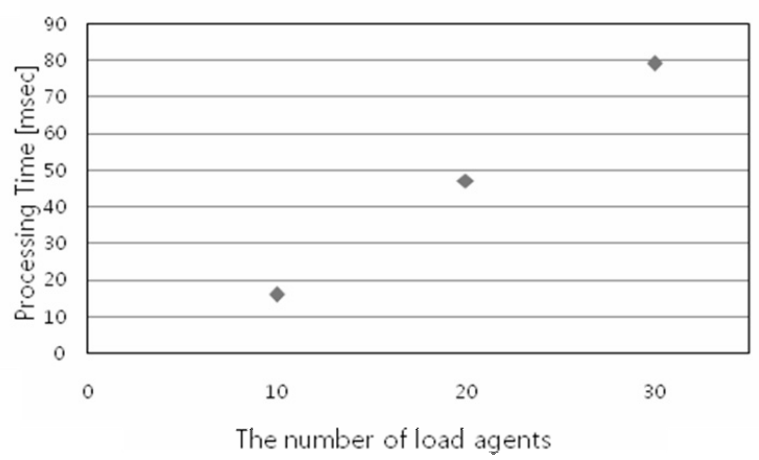

Fig. 14. Processing time according to the number of load agents.

Table 3. DG information

\begin{tabular}{c|c|c}
\hline DG & Production cost $(\notin / \mathrm{kWh})$ & Capacity $(\mathrm{kWh})$ \\
\hline DG1 & 70 & 10 \\
\hline DG2 & 80 & 30 \\
\hline DG3 & 90 & 40 \\
\hline DG4 & 95 & 50 \\
\hline
\end{tabular}

Table 4. DS information

\begin{tabular}{c|c|c}
\hline DS & \multicolumn{2}{|c}{ Information $(\mathrm{kWh})$} \\
\hline DS1 & Charged: 6 & Available: 4 \\
\hline DS2 & Charged: 3 & Available: 2 \\
\hline
\end{tabular}

termined by the processing algorithms of agents; for instance, in Step 4, the iterative algorithm based on the Talmud rule is adopted in the MGOCC agent. The time complexities of these algorithms are proportional with the number of agents (i.e., linear order); hence, the total processing time of the proposed method is in linear order. To confirm the time complexity of the proposed method, Test 2 was conducted for three cases using 10, 20, and 30 load agents. Table 2 shows the number of agents of the three cases. The variable currentTimeMillis(), which is a method in Java, was used to measure the processing time for solving load shedding. Fig. 14 shows the result of Test 2, confirming that the processing time has a linear relation with the number of load agents.

\subsection{Test 3}

In Test 3, establishing a new operation plan by a sudden fault of one DG (DG2) after deciding an operation plan for the next interval is considered as mentioned before. Here, 


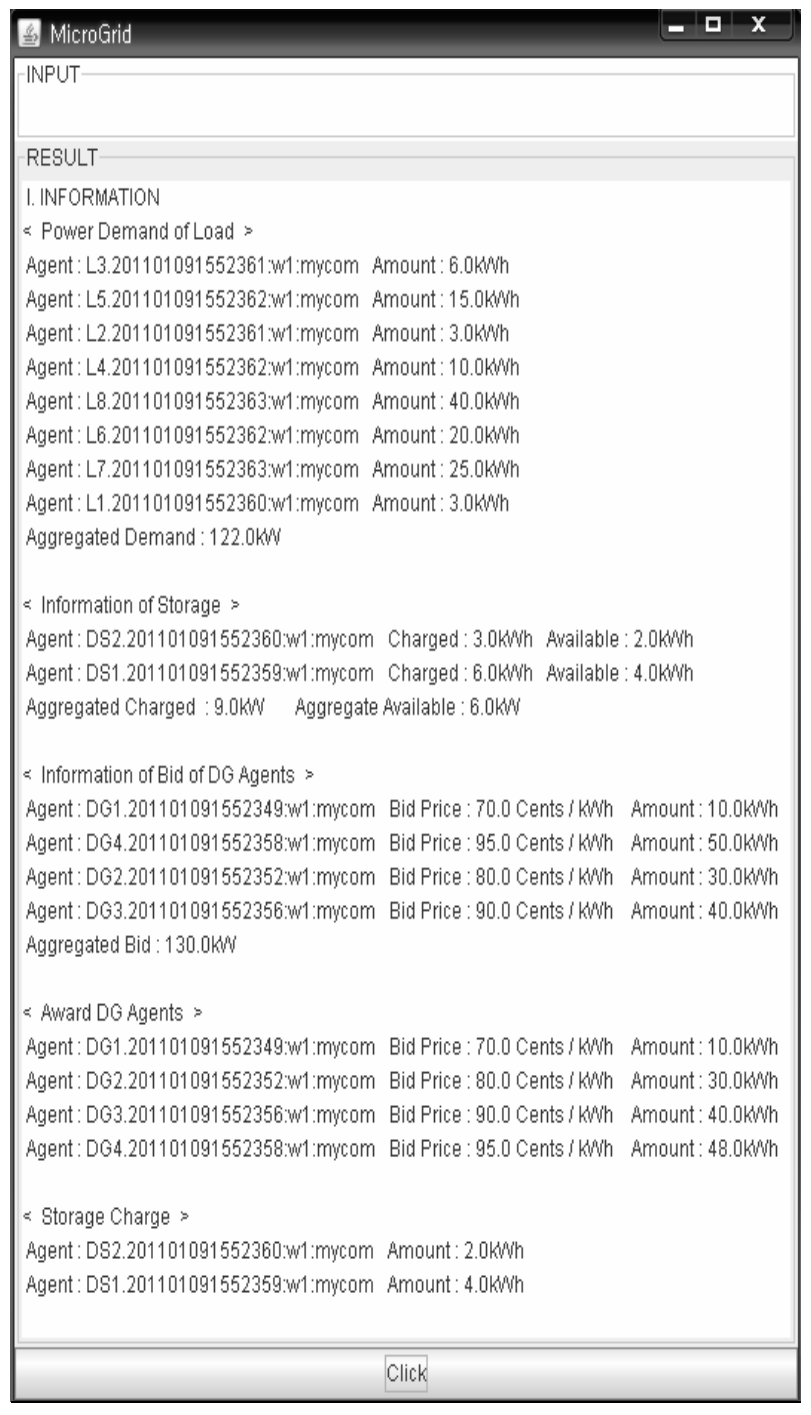

Fig. 15. Operation plan before a sudden fault of DG2.

load shedding based on the Talmud rule is used to solve a power imbalance (i.e., supply shortage). Details for islanded microgrid operation based on the multiagent system are described in our previous work [17].

Table 3 shows production costs and capacities of four DGs and Table 4 shows information of two DSs, respectively.

Eight loads are considered:

- L1: $3 \mathrm{kWh}$

- L2: $3 \mathrm{kWh}$

- L3: $6 \mathrm{kWh}$

- L4: $10 \mathrm{kWh}$

- L5: $15 \mathrm{kWh}$

- L6: $20 \mathrm{kWh}$

- L7: $25 \mathrm{kWh}$

- L8: $40 \mathrm{kWh}$

Fig. 15 shows an operation plan decided by agents for the next interval before a sudden fault of DG2. Here, two DSs are charged because of supply surplus and output of

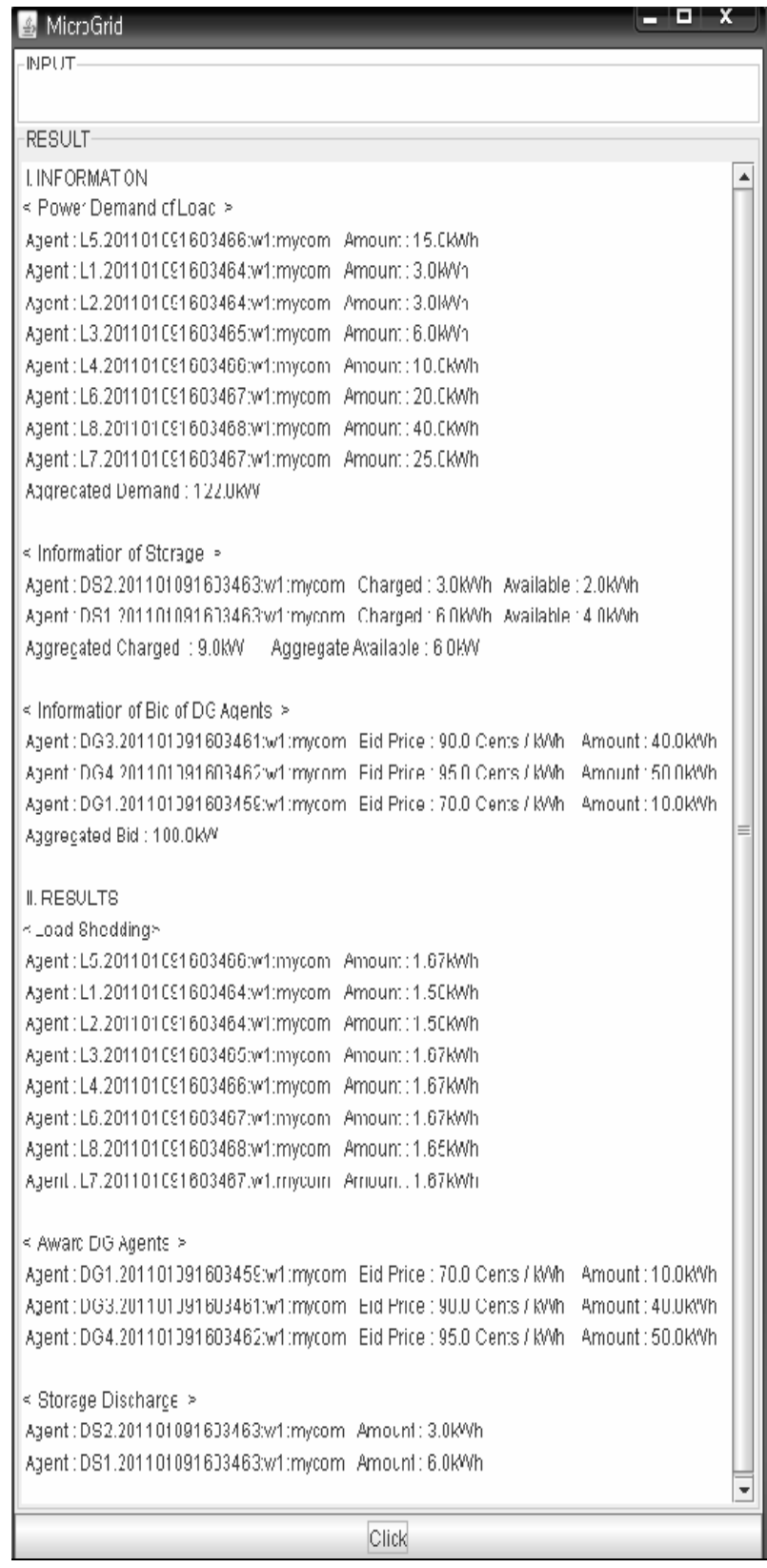

Fig. 16. Operation plan after a sudden fault of DG2.

DG4 is decreased by the merit order as mentioned in Section 4 because it has the highest bidding price.

Fig. 16 shows a new operation plan established to solve the emergency (i.e., supply shortage after the sudden fault of DG2). From the results, all DGs and all DSs supply power to solve the supply shortage. In addition, the proposed load-shedding scheme is performed. Here, L8 has amounts below the decimal point by the computation rule mentioned in Section 3.

\subsection{Discussion}

The Talmudic pattern of load shedding in the proposed agent-based microgrid operation with 10 load agents is shown in Fig. 13. Power imbalance between supply and 
demand in the real microgrid is minimal because total generation capacity is decided by the total load amounts in a microgrid. In other words, the load-shedding area is anticipated to be between 0 and $300 \mathrm{kWh}$ (Fig. 13). Therefore, an equal amount of load shedding is applied to every load when supply shortage occurs. However, relatively large load shedding is performed near proportional to the amount of load or by large loads during a contingency (Fig. 13).

The proposed load-shedding scheme provides a unique solution regardless of the number of load agents because it is based on the Talmud rule. Furthermore, the processing time for solving a load-shedding problem is proportional to the number of load agents. Approximately $270 \mathrm{~ms}$ is predicted for load shedding in islanded microgrid operation with 100 loads. The proposed load-shedding scheme is therefore acceptable for application in various fields.

In addition, the proposed multiagent system is able to operate the islanded microgrid regardless of imbalance operation conditions (i.e., supply surplus and shortage from Test 3).

If critical or important loads having the high priority are considered in practical applications, the suggested load scheme should be applied to the rest of the loads excluding the critical or important loads.

\section{Conclusion}

In this paper, a load-shedding scheme using the Talmud rule in autonomous operation based on a multiagent system for an islanded microgrid is presented. The proposed scheme was successfully performed in agent-based microgrid operation. The contributions of this paper are summarized as follows:

- The load-shedding problem was approached as a bankruptcy problem.

- The load-shedding problem was modeled on a bankruptcy problem, and a load-shedding scheme was established based on the Talmud rule.

- The proposed load-shedding scheme was successfully tested to a multiagent system for islanded microgrid operation.

- The processing time of the proposed load-shedding scheme was acceptable for real application.

In the future, we plan to study other detailed schemes required to apply a multiagent system to microgrid operation.

Note: This is an extended version of our SUcomS 2010 paper [18].

\section{References}

[1] A. L. Dimeas, and N. D. Hatziargyriou, "Operation of a Multiagent System for Microgrid Control," IEEE
Trans. on Power Systems, Vol. 20, No. 3, pp.14471455, Aug. 2005.

[2] A. L. Dimeas, and N. D. Hatziargyriou, "A Multiagent System for Microgrids", 2004 IEEE Power Engineering Society General Meeting, pp. 55-58 June 2004

[3] H.-M. Kim, and T. Kinoshita, "A Multiagent System for Microgrid Operation in the Grid-interconnected Mode," Journal of Electrical Engineering \& Technology, Vol. 5, No. 2, pp. 246-254, June 2010.

[4] H.-M. Kim, and T. Kinoshita, "Multiagent System for Microgrid Operation based on a Power Market Environment," INTELEC 2009, Incheon, Korea, Oct. 2009.

[5] M. Wooldridge, An Introduction to Multiagent Systems, $2^{\text {nd }}$ Edition, A John Wiley and Sons, Ltd, Publication, 2009.

[6] R. J. Aumann, and M. Michael, "Game theoretic analysis of a bankruptcy problem from the Talmud," Journal of economic theory, Vol. 36, pp. 195-213, 1985.

[7] J. D. Moreno-Ternero, and A. Villar, "The Talmud rule and the securement of agents' awards", Mathematical Social Sciences, pp.245-257, 2004

[8] H. Yaiche, R. R. Mazumdar, and C. Rosenberg, "A game theoretic framework for bandwidth allocation and pricing in broadband networks," IEEE/ACM Trans. on networking, Vol. 8, No. 5, pp. 667-678, 2000.

[9] W. Thomson, "Axiomatic and game-theoretic analysis of bankruptcy and taxation problems: a survey," Mathematical social science, Vol. 45, pp. 249-297, 2003.

[10] S. Russell and P. Norvig, Artificial Intelligence - A Modern Approach, $3^{\text {rd }}$ Edition, Pearson, 2010.

[11] T. Kinoshita (Ed.), Building Agent-based Systems, The Institute of Electronics, Information and Communication Engineers (IEICE), Japan, 2001. (in Japanese)

[12] R. G. Smith, "The Contract Net Protocol: High-level Communication and Control in a Distributed Problem Solver," IEEE Trans. on Computer, Vol. C-29, No. 12, pp. 1104-1113, Dec. 1980.

[13] G Weiss, editor, Multiagent Systems: A Modern Approach to Distributed Artificial Intelligence, The MIT press, 1999.

[14] T. Kinoshita, and K. Sugawara, “ADIPS Framework for Flexible Distributed Systems," T. Ishide (ed.), Multiagent Platforms, LNAI 1599, pp. 18-32, 1998.

[15] T. Uchiya, T. Maemura, L. Xiaolu, and T. Kinoshita, "Design and Implementation of Interactive Design Environment of Agent System," Proc. of $20^{\text {th }}$ Int. Conf. Industrial, Engineering and Other Applications of Applied Intelligent Systems (IEA/AIE 2007), LNAI4570, AAAI/ACM, pp. 1088-1097, 2007.

[16] IDEA/DASH Tutorial [Online]. Available: http:// www.ka.riec.tohoku. ac.jp/idea/index.html.

[17] H.-M. Kim, T. Kinoshita, and M.-C. Shin, A Multi- 
agent System for Autonomous Operation of Islanded Microgrid based on a Power Market Environment, Energies, Vol. 3, Issue 12, pp. 1972-1990, Dec. 2010.

[18] H.-M. Kim, T. Kinoshita, Y. Lim, and T.-H. Kim "Talmud Rule Approach to Load-shedding in Agentbased Microgrid Operation", In T.-H. Kim, A. Stoica, R.-S. Chang (Eds), Sercurity-Enriched Urban Computing and Smart Grid, Communications in Computer and Information Science, Vol. 78, pp. 621-628, Springer-Verlag Berlin Heidelberg, SUcomS 2010, Daejeon, Korea, Sep. 2010.

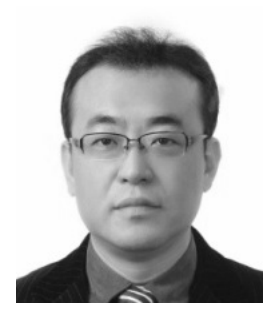

Hak-Man Kim received his B.S., M.S., and Ph.D. degrees in Electrical Engineering from Sungkyunkwan University, Korea, in 1991, 1993, and 1998, respectively, as well as his second $\mathrm{Ph} . \mathrm{D}$. degree in Computer and Mathematical Sciences from Tohoku University, Japan, in 2011. He was a senior researcher in the Korea Electrotechnology Research Institute from 1996 to 2008. Currently, he is a professor in the Department of Electrical Engineering, University of Incheon, Korea. His research interests include power system analysis and modeling, multiagent-based intelligent microgrids and smart grids, and agent engineering. He is a senior member of KIEE, and is a member of IEEE, and IEICE.

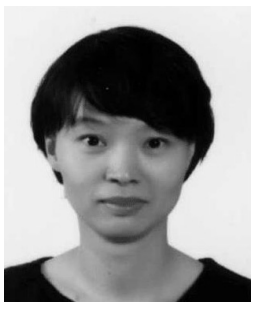

Yujin Lim received her B.S., M.S., and $\mathrm{Ph} . \mathrm{D}$. degrees in Computer Science from Sookmyung Women's University, Korea, in 1995, 1997, and 2000 , respectively. Since 2004, she has been an assistant professor in the Department of Information Media, University of Suwon. Her research interests include sensor networks, mesh networks, vehicular ad hoc networks, and smart grids.

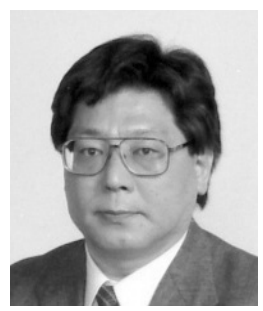

Tetsuo Kinoshita is a professor of Research Institute of Electrical Communication of Tohoku University. He received his B.E. degree in electronic engineering from Ibaraki University, Japan, in 1977, and his M.E. and Dr.Eng. degrees in Information Engineering from Tohoku University, Japan, in 1979 and 1993, respectively. His research interests include agent engineering, knowledge engineering, and knowledge-based/agent-based systems. Dr. Kinoshita received the IPSJ Research Award, the IPSJ Best Paper Award, and the IEICE Achievement Award in 1989, 1997, and 2001, respectively. In addition, he is a fellow of IEICE and IPSJ, and is a member of IEEE, ACM, AAAI, JSAI, and the Society for Cognitive Science of Japan. 